Reprod. Nutr. Dévelop., 1987, 27 (1 B), 275-276.

\title{
Effet du jeûne et de la fréquence de distribution des repas sur les variations de l'urémie et du taux d'urée du lait chez la chèvre laitière
}

J. BRUN-BELLUT, N. ABDUL-AHAD, B. VIGNON

Zootechnie, E.N.S.A.I.A.,

2, Av. de la Foret de Haye,

B.P. 172, 54505 Vandceuvre-les-Nancy Cedex.

Summary. Four lactating goats were used in a $4 \times 4$ Latin square experiment to determine the effect of feeding frequency ( 2 and 6 times daily) and starvation on diurnal variations of uremia and the milk urea level (TUL). Frequent feeding did not change the average daily uremia and TUL ; but lowered diurnal variation. Starvation increased uremia and TUL.

L'urémie est considérée comme un indicateur de l'état de nutrition azotée des ruminants (Harmeyer et Martens, 1980). Le taux d'urée du lait (TUL), qui est fortement corrélé à l'urémie, est aussi utilisé comme indicateur chez les animaux en lactation: vaches (Journet et al., 1975) et chèvres (Brun-Bellut et al., 1983). L'urémie n'étant souvent mesurée qu'une fois dans la journée, nous avons étudié ses variations nychtémérales, ainsi que celles dues aux effets de la fréquence des repas et du jeûne.

Matériel et méthodes. Quatre chèvres en lactation (4e semaine) sont utilisées pendant 4 semaines. Les animaux reçoivent chacun une ration calculée pour couvrir les besoins d'entretien et de production mesurés la semaine précédant l'expérimentation. Aussi, d'une chèvre à l'autre, les rations varient entre 900 et $1100 \mathrm{~g}$ de pulpes de betteraves déshydratées, 700 et $1000 \mathrm{~g}$ d'orge, 50 et $100 \mathrm{~g}$ de tourteaux de soja, 20 et $22 \mathrm{~g}$ d'urée. La ration établie en début de période pour chaque chèvre n'est pas modifiée pendant les 4 semaines d'expérimentation. Les digestibilités et les bilans azotés sont mesurés par période de 5 jours consécutifs. Le $6^{e}$ jour est réservé aux prélèvements - lait, urine (par sondes), sang (veine jugulaire) - qui sont effectués toutes les 3 heures. Les mesures du $6^{e}$ et $7^{\circ}$ jour ne sont pas utilisées dans le calcul des bilans azotés.

Protocole : répartition du nombre de distributions quotidiennes d'aliments.

\begin{tabular}{cllll}
\hline Semaines & 1 & 2 & 3 & 4 \\
\hline Chèvres : & 2 & $2^{*}$ & 6 & $6^{*}$ \\
1 & 6 & $6^{*}$ & 2 & $2^{*}$ \\
2 & $2^{*}$ & 2 & $6^{*}$ & 6 \\
3 & $6^{*}$ & 6 & $2^{*}$ & 2 \\
\hline
\end{tabular}

* chèvres à jeun le jour de prélèvement.

Résultats (tabl. 1, fig. 1). Les rations prévues par le protocole sont toujours consommées en quasi-totalité $(<5 \%$ de refus). La digestibilité des matières azotées, systématiquement supérieure quand les animaux reçoivent 6 repas $(+0,04)$, témoigne d'une meilleure dégradation et digestion intestinale des protéines alimentaires peut être liée à un transit moyen plus lent. La rétention azotée plus élevée dans le cas de 6 distributions d'aliments pourrait témoigner d'une amélioration de l'utilisation de l'azote très fermentescible dans le rumen. L'augmentation de l'apport d'azote digéré dans l'intestin et la diminution des pertes d'azote fermentescible dans le rumen dans le cas de 6 repas expliquent, pour des animaux en bilan azoté positif, que l'excrétion urinaire d'azote et le TUL ne soient pas différents dans les deux cas. 
TABL. 1. - Consommation, digestion et utilisation des aliments.

\begin{tabular}{|c|c|c|c|c|c|c|c|c|c|}
\hline & & \multicolumn{2}{|c|}{ Matière sèche } & & \multicolumn{2}{|c|}{ Digestibilité } & \multirow[b]{2}{*}{$\begin{array}{l}\text { Bilan } \\
\text { azoté } \\
\text { (g) }\end{array}$} & \multicolumn{2}{|c|}{ Quantité produite/jour } \\
\hline & & $\begin{array}{c}\text { proposée } \\
\text { (g) }\end{array}$ & $\begin{array}{l}\text { ingérée } \\
\text { (g) }\end{array}$ & $\begin{array}{c}\text { Taux MAT } \\
\text { ration } \\
\%\end{array}$ & MO & MAT & & $\begin{array}{c}\text { Lait } \\
3,5 \% \mathrm{MG} \\
(\mathrm{kg})\end{array}$ & $\begin{array}{c}\text { Azote } \\
\text { (g) }\end{array}$ \\
\hline 2 repas & $\begin{array}{l}\mathrm{m} \\
\sigma\end{array}$ & $\begin{array}{r}2030 \\
189\end{array}$ & $\begin{array}{r}1962 \\
275\end{array}$ & $\begin{array}{r}13,1 \\
1,0\end{array}$ & $\begin{array}{l}0,76 \\
0,05\end{array}$ & $\begin{array}{l}0,70 \\
0,04\end{array}$ & $\begin{array}{l}4,4 \\
3,2\end{array}$ & $\begin{array}{l}2,90 \\
0,30\end{array}$ & $\begin{array}{r}13,68 \\
1,35\end{array}$ \\
\hline 6 repas & $\begin{array}{l}\mathrm{m} \\
\sigma\end{array}$ & $\begin{array}{r}2038 \\
127\end{array}$ & $\begin{array}{r}1928 \\
247\end{array}$ & $\begin{array}{r}13,2 \\
0,9\end{array}$ & $\begin{array}{l}0,77 \\
0,03\end{array}$ & $\begin{array}{l}0,74 \\
0,04\end{array}$ & $\begin{array}{l}6,6 \\
4,2\end{array}$ & $\begin{array}{l}2,86 \\
0,44\end{array}$ & $\begin{array}{r}12,79 \\
1,53\end{array}$ \\
\hline
\end{tabular}
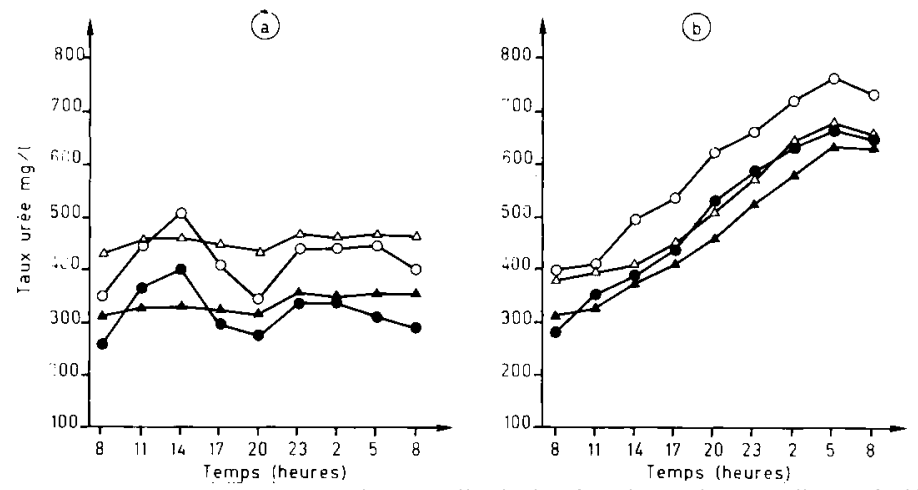

FIG. 1. - Evolution du taux d'urée du lait et de l'urémie chez les animaux alimentés (1-a) et chez les animaux à jeun (1-b).

- 1a. Lait: $-\Delta-6$ distributions; --2 distributions. Sang: $-\Delta-6$ distributions ; - -2 distributions. $-1 b$. Lait : $-\triangle-$ Animaux recevant précédemment 6 repas ; $-0-$ Animaux recevant précédemment 2 repas. Sang : $-\mathbf{\Delta}-$ Animaux recevant précédemment 6 repas ; - - Animaux recevant précédemment 2 repas.

Les variations nycthémérales de l'urémie sont beaucoup plus importantes avec 2 distributions qu'avec 6 (coefficients de variations : 14 et $5 \%$ ). Cependant, les taux moyens quotidiens ne sont pas différents entre les 2 rythmes de distribution (324 et $334 \mathrm{mg} / \mathrm{l}$ ). L'urémie des animaux maintenus à jeun augmente régulièrement pendant $24 \mathrm{~h}$ et est multipliée par deux. Le TUL mesuré à chaque prélèvement suit la même évolution que l'urémie, mais lui est toujours supérieur, que les animaux soient à jeun ou qu'ils reçoivent 2 à 6 distributions d'aliments : TUL = 0,86 urémie $+149 r=0,92 \mathrm{n}=144$. Cette différence déjà observée dans d'autres expériences (Abdul Ahad, 1986) correspond à l'hypothèse émise par Linzell et Peaker (1974) qui indiquent que le passage de l'urée du lait au sang est plus lent que le passage inverse.

Les variations nycthémérales importantes de l'urémie quand l'alimentation est peu fractionnée limitent son emploi comme indicateur du métabolisme azoté. Urémie et TUL moyens quotidiens ne permettent pas de mettre en évidence la meilleure digestion et la meilleure rétention azotée obtenues dans le cas de 6 repas ; d'autres indicateurs devront être utilisés pour permettre d'apprécier ces modifications qui ont des effets opposés sur l'évolution de l'urémie et de TUL.

Abdul-Ahad N., 1986. Thèse I.N.P.L. Nancy.

Brun-Bellut J., Laurent F., Vignon B., 1983. IVe Symp. Int. Métabolisme et Nutrition azotés.

Clermont-Ferrand (France), 3-9, 1983. Ed. I.N.R.A. Publ., II.

Harmeyer J., Martens H., 1980. J. Dairy Sci., 63, 1707-1728.

Journet M., Vérité R., Vignon B., 1975. Le Lait, 54, 3-4, 212-223.

Linzell J. L., Peaker M., 1974. J. Physiol., 243, 129-151. 